\title{
Establishment and Evaluation of Immune Checkpoint Inhibitor Treatment Response Model Based on Gene Expression Value
}

\section{Xiaoyan Li}

Shanxi Provincial People's Hospital

\section{Guoyin Li}

Zhoukou Normal University

\section{Xiyang Tang}

Tangdu Hospital, Fourth Military Medical University

\section{Yongsheng Zhou}

Tangdu Hospital, Fourth Military Medical University

\section{Kaifu Zheng}

Tangdu Hospital, Fourth Military Medical University

\section{Zhonglin Luo}

Peace Hospital affiliated to Changzhi Medical College囚Changzhi Medical College

\section{Xiao Zhang}

Fourth Military Medical University

\section{Yanlu Xiong}

Tangdu Hospital, Fourth Military Medical University

Yangbo Feng ( $\sim$ fengyb_thoracic@163.com )

Tangdu Hospital, Fourth Military Medical University

\section{Research Article}

Keywords: Immune checkpoint, immunotherapy, melanoma, model, programmed cell death 1

Posted Date: September 13th, 2021

DOl: https://doi.org/10.21203/rs.3.rs-870917/v1

License: (c) (i) This work is licensed under a Creative Commons Attribution 4.0 International License. Read Full License 


\section{Abstract}

Background: In the past 10 years, the identification of new mutant genes involved in the pathogenesis of melanoma and the discovery of key immune checkpoints have promoted the development of targeted therapy and immunotherapy. There is no doubt that an important breakthrough has been made in the treatment of advanced or metastatic melanoma. However, the treatment of melanoma also faces many challenges. In addition to resistance to existing targeted therapies or immunotherapy, most patients do not respond to immunotherapy or have serious adverse reactions. At present, the value of existing biomarkers to predict treatment response and toxicity is still limited. Therefore, there is an urgent need to establish a convenient and reliable immunotherapy response prediction model in order to preliminarily clarify the population benefiting from immunotherapy.

Results: We established a predictive model based on the expression values of five genes for patients with melanoma with an anti-PD1 immunotherapy response score. This model showed better predictive ability compared with other common immunotherapy predictors. Differences were found in the number of immune cells and the expression of common immune checkpoint genes between the high- and low-score groups. The model played a pivotal role in predicting renal cell carcinoma anti-PD1 immunotherapy response.

Conclusions: The anti-PD1 immunotherapy response score prediction model for patients with melanoma showed good predictive power, thus having far-reaching significance for identifying people who benefited from anti-PD1 immunotherapy and reducing the potential toxicity of insensitive patients.

\section{Background}

Melanoma is a tumor with a high degree of malignancy derived from melanocytes; it mainly occurs in the skin, mucous membranes, and uveal tract of the eye. In 2015, the global incidence of melanoma was 351,880 cases, and the age-standardized incidence rate was 5 per 100,000 persons. At the same time, it caused 59,782 deaths worldwide, with an age-standardized rate of 1 death per 100,000 persons ${ }^{[1]}$. In the United States, the incidence of cutaneous melanoma increases every year, but the mortality rate has dropped significantly ${ }^{[2]}$. In 2017, the incidence of melanoma in China was estimated to be 16,073 cases, and the age-standardized rate was 0.9 cases per 100,000 people. The death toll due to melanoma was estimated to be 5088 , and the age-standardized ratio was 0.3 per $100,000^{[3]}$. Melanoma is a relatively rare malignant tumor in China; however, the incidence rate shows an increase and the mortality rate is high ${ }^{[4]}$. This difference is mainly due to the different common subtypes of melanoma in China and the United States. Chinese melanoma is more malignant. Among Asians and other people of color, melanomas of the extremities and mucous membranes are more common, while melanomas of whites' skin are more common.

Surgical treatment is the main choice for more than $90 \%$ of primary focal melanomas ${ }^{[5]}$. After early melanoma is diagnosed, wide excision of the primary tumor with safe margins should be performed 
based on the depth of tumor invasion (Breslow thickness). Most of these tumors can be cured by surgery. It is generally believed that melanoma is not sensitive to radiotherapy. However, radiotherapy can shrink the tumor and relieve symptoms in cases of brain metastasis, bone metastasis, and repeated lymph node recurrence. Patients do not benefit much from single-agent chemotherapy or traditional combination drugs; the effective rate is about $10-15 \%[4]$. In the last 10 years, the identification of new mutant genes involved in the pathogenesis of melanoma and the discovery of key immune checkpoints have promoted the use of targeted therapy and immunotherapy. An important breakthrough has been made in treating advanced or metastatic melanoma ${ }^{[6,7]}$. About $50 \%$ of patients with metastatic melanoma treated with combined immunotherapy survive for 5 years after diagnosis ${ }^{[8]}$. In addition, the 5 -year survival rate of patients with targeted therapy combined with BRAF/ mitogen activated protein kinase (MAPK) kinase or single-agent PD1 blockade is more than one third ${ }^{[9,10]}$. Despite these advances, the current cure rate for patients with metastatic melanoma remains low. In summary, the treatment of melanoma still faces many challenges. Besides drug resistance to existing targeted therapy or immunotherapy, most patients do not respond to immunotherapy or have serious adverse reactions, which is also worth further consideration $^{[11]}$. Elucidating the determinants of immunotherapy response and drug resistance is the key to improving patient prognosis and developing new treatment strategies. Currently, the value of existing biomarkers to predict therapeutic response and toxicity is still limited ${ }^{[5,6]}$. Therefore, a convenient and reliable immunotherapy response prediction model needs to be urgently established to initially determine which patients are most likely to benefit from immunotherapy.

In this study, we obtained melanoma RNA sequencing datasets containing anti-programmed cell death 1 (anti-PD1) immunotherapy response information from the Gene Expression Omnibus (GEO) database, screened out the differentially expressed genes between the anti-PD1 immunotherapy response group and the nonresponse group, and established a key gene-based predictive model of anti-PD1 immunotherapy response to melanoma. Subsequently, the accuracy of the model was evaluated, and the efficacy of this model and common indicators of immunotherapy response was compared. Further, the relationship between the model and the tumor microenvironment was analyzed, and the application value of this model in other tumors was evaluated. The findings of this study might provide an important reference for the reasonable selection of treatment options for patients with melanoma and avoid unnecessary potential toxicity.

\section{Results}

\section{Construction of the predictive model of anti-PD1 immunotherapy response}

Differentially expressed genes play an important role in the occurrence and development of tumors, and hence the identification of these genes can help deepen our understanding of tumors. First, we obtained RNA sequencing datasets (GSE78220 and GSE91061) containing anti-PD1 treatment response information from the GEO database (Table 1). The aforementioned datasets were merged into a new 
dataset for subsequent analysis through batch correction. We obtained 1874 differentially expressed genes through differential expression analysis, including 1097 upregulated genes and 777 downregulated genes (Fig. 1A). Next, we randomly divided the new dataset into training and validation datasets (the ratio of sample size was 6:4). We obtained five key genetic variables using the "glmnet" package to perform Lasso regression and cross-validation, and constructed a binary logistic regression model for the training dataset (the response to immunotherapy was recorded as 0 , and the nonresponse was recorded as 1 ) (Fig. 1B). Therefore, the lower the score, the more sensitive the immunotherapy response. This model mainly predicted the anti-PD1 immunotherapy response of patients with melanoma. The key genes involved in this model were protein tyrosine phosphatase domain containing 1 (PTPDC1), serologically defined colon cancer antigen 8 (SDCCAG8), Peter Pan Homolog (PPAN), serine/threonine kinase 40 (STK40), and small nucleolar RNA, C/D box 16 (SNORD16) (Table 2). Finally, the anti-PD1 immunotherapy response of each tumor sample was predicted using the predict() function. We revealed the relationship between the actual immunotherapy response of tumor samples and the immunotherapy response score using differential expression analysis. The results showed that the actual immunotherapy nonresponse group had a significantly higher score than the response group (Fig. 1C). This finding illustrated that the score clearly distinguished the immunotherapy response group from the nonresponse group, which was of great significance for predicting the immunotherapy response.

\section{Evaluation and verification of the anti-PD1 immunotherapy response prediction model}

As mentioned earlier, we constructed an anti-PD1 immunotherapy response prediction model, but the accuracy of this model needed further evaluation. Based on the constructed model, we calculated the immunotherapy response score of the validation dataset. This score clearly distinguished the immunotherapy response group from the nonresponse group in the validation dataset (Fig. 1D). The ROC curve analysis showed that the area under the curve (AUC) of the immunotherapy response score prediction model based on the training dataset was 0.972 (Fig. 1E). In this model, sensitivity (SE), specificity (SP), positive predictive value (PPV), negative predictive value (NPV), and accuracy were 1 , $0.708,0.889,1$, and 0.913 , respectively (Table 3 ). Subsequently, we validated the model with the validation dataset. The AUC of the immunotherapy response score prediction model based on the validation dataset was 0.797 (Fig. 1E). The SE, SP, PPV, NPV, and accuracy of this model was 0.923 , $0.571,0.857,0.727$, and 0.830 , respectively (Table 3 ). This suggested that the predictive effect of the immunotherapy response prediction model for patients with melanoma was good.

\section{Comparison of the anti-PD1 immunotherapy response prediction model with other indicators}

At present, the common predictors of immunotherapy efficacy include PDL1 expression, tumor mutation burden, deficient mismatch repair (dMMR), and so forth ${ }^{[12]}$. We evaluated the role of the aforementioned 
indicators in predicting the response to anti-PD1 immunotherapy to compare the effectiveness of the model and other common indicators. DNA mismatch repair is a highly conservative process involving four key genes mutL homologue 1 (MLH1), postmeiotic segregation increased 2 (PMS2), mutS homologue $2(\mathrm{MSH} 2)$, and mutS homologue $6(\mathrm{MSH} 6)^{[13]}$. We used PCA to obtain an algorithm that reflected the dMMR process based on the expression values of the aforementioned four genes (Fig. $2 \mathrm{~A}$ and $2 \mathrm{~B}$ ). Subsequently, the algorithm's role in predicting the response of patients with melanoma to antiPD1 immunotherapy was evaluated. The results showed that the AUC of the dMMR algorithm in the training and validation datasets was 0.615 and 0.473 , respectively (Fig. $2 \mathrm{C}$ and $2 \mathrm{D}$ ). The ROC curve analysis showed that the AUC of the PDL1 expression in the training and validation datasets was 0.606 and 0.538 , respectively (Fig. $2 \mathrm{E}$ and $2 \mathrm{~F}$ ). The accuracy of the aforementioned common predictive indicators was lower than that of the constructed model, which showed that the model had certain advantages and value in predicting the response of immunotherapy.

\section{Correlation between the anti-PD1 immunotherapy response prediction model and tumor microenvironment}

The tumor microenvironment is mainly composed of stromal cells and recruited immune cells. We used the "estimate" algorithm to estimate the number of immune and matrix components in the melanoma samples. We used the Wilcoxon test to perform differential expression analysis so as to reveal the correlation between the ratio of matrix and immune components in tumor samples and the score of immunotherapy response. The results showed that samples with a higher immunotherapy response score had a lower matrix score $(P=0.019)$ and a lower immune score $(P<0.001)$ (Fig. 3A and 3B). This indicated that tumor samples that were more sensitive to anti-PD1 immunotherapy had higher levels of stromal and immune components.

Subsequently, we performed GSEA on melanoma samples from the groups with high $(n=66)$ and low ( $n$ $=67$ ) immunotherapy response scores (Fig. 3C, 3D, 3E and 3F). The results showed that melanomas in the low-score group were significantly enriched in the biological processes of allograft rejection, antigen binding, B-cell receptor signaling pathway, interleukin 4 production, T-cell selection, dendritic cell pathway, Th1/Th2 pathway, and natural killer T cells pathway. Obviously, the lower the anti-PD1 immunotherapy score, the better the immunotherapy effect. This phenomenon might be attributed to the activation of part of the tumor's immune function.

To reveal the correlation between immunotherapy response score and TICs, we used the CIBERSORT algorithm to analyze the proportion of infiltrating immune subpopulations in melanoma tissues and constructed the expression profiles of 22 immune cells in these samples (Fig. 4A). We explored the relationship between immune cells using Pearson correlation analysis (Fig. 4B). Next, we assessed whether a difference existed in the expression of immune cells between the high and low scores of immunotherapy responses. The analysis showed differences in the naive B cells, CD $8+T$ cells, gamma/delta T cells, monocytes, and plasma cells in the samples of patients with melanoma between 
the high- and low-score groups of anti-PD1 immunotherapy response $(P<0.05)$ (Fig. 4C). As shown in the previous correlation analysis of immune cells, CD8 $+T$ cells and gamma/delta $T$ cells were positively correlated. Subsequently, we discussed the expression characteristics of common immune checkpoint genes based on the high and low scores of immunotherapy responses. We found that most immune checkpoint genes were highly expressed in patients with melanoma in the low-score group $(P<0.05)$ (Fig. 4D). This indicated that the more sensitive the patient's anti-PD1 immunotherapy, the higher the expression of immune checkpoint genes.

\section{Value of immunotherapy response scores in other tumors based on the model}

Although our model played a significant role in predicting the response of patients with melanoma to anti-PD1 immunotherapy, whether this model was applicable to other tumors was not clear. We obtained the renal cell carcinoma dataset GSE67501 containing anti-PD1 immunotherapy response information from the GEO database to disclose the role of this model in other tumors. We scored the immunotherapy response to renal cell carcinoma based on the established model algorithm. The ROC curve analysis showed that the AUC of the immunotherapy response score in this dataset was 0.679 (Fig. 4E). Despite no significant difference in the immunotherapy scores between the actual immunotherapy responsesensitive and insensitive groups, the immunotherapy response scores in the immunotherapy-sensitive groups showed a lower trend, which was consistent with our previous results (Fig. 4F). This suggested that our model played a role in predicting renal cell carcinoma immunotherapy response, which provided a reference for selecting immunotherapy options for patients with renal cell carcinoma.

\section{Discussion}

Melanoma is one of the most aggressive malignant tumors, and its incidence has increased significantly over the past decade ${ }^{[14]}$. Despite the remarkable progress made in treating melanoma in recent years, most patients with metastatic melanoma cannot be cured. A large proportion of patients do not respond well to immunotherapy or have serious adverse reactions ${ }^{[11]}$. Obviously, the existing indicators that reflect the response of immunotherapy are not enough to cope with the clinical decision-making of patients. Therefore, an accurate and feasible immunotherapy response prediction model needs to be established. Establishing various tumor large-scale databases and developing current omics technology provide theoretical and technical support for establishing immunotherapy response prediction models based on gene expression values ${ }^{[15,16]}$.

In this study, we used the RNA sequencing data of samples from the anti-PD1 immunotherapy response group and nonresponse group to perform differential expression analysis and obtained 1097 upregulated genes and 777 downregulated genes. Subsequently, the dataset was randomly divided into training and validation datasets. The immunotherapy response score prediction model based on the training dataset containing multiple genetic variables (PTPDC1, SDCCAG8, PPAN, STK40, and SNORD16) was 
constructed using the "glmnet" package and verified in the validation dataset. The results showed that the AUC value of the immunotherapy response score prediction models of the training and validation datasets was 0.9717 and 0.7967 , respectively. Next, we compared the model with the current common immunotherapy predictors. The AUC value of PDL1 expression in the training and validation datasets was 0.606 and 0.538 , respectively. The AUC values of PD1 expression in the training and validation datasets was 0.726 and 0.595 , respectively. The AUC value of the dMMR algorithm in the training and validation datasets was 0.615 and 0.473 , respectively. This demonstrated that the model showed certain advantages in predicting the response to immunotherapy. In addition, we found that tumor samples with better response to anti-PD1 immunotherapy had higher levels of stromal and immune components. The GSEA analysis indicated that the obvious immunotherapeutic effect might be due to the activation of part of the tumor immune function. We assessed the correlation between the model and TICs and common immune checkpoint genes. The results suggested that some immune cells and immune checkpoint genes were different between the high and low immunotherapy response scores. Finally, we evaluated the role of this model in predicting immunotherapy response in the renal cell carcinoma dataset. The AUC value of the immunotherapy response score of this dataset was 0.679 , which reflected the contribution of this model in predicting the renal cell carcinoma immunotherapy response.

In fact, the genes involved in this model have important biological significance, especially in tumor transformation and malignant progression. The protein encoded by the PTPDC1 gene is a cell cyclerelated phosphatase involved in centrosome replication and cytoplasmic division ${ }^{[17]}$. The SDCCAG8 gene is a centrosome protein related to nephronophthisis-related ciliopathies, which is expressed in kidney and lung epithelial cells. This gene is involved in the $\mathrm{S}$ phase of the cell cycle, and its deletion can lead to abnormal activation of the DNA damage response related to replication stress ${ }^{[18]}$. In addition, studies have shown that SDCCAG8 is related to cell proliferation, migration, and invasion of head and neck squamous cell carcinoma ${ }^{[19]}$. The PPAN gene was originally discovered in the screening of mutants with growth defects in Drosophila. The protein encoded by this gene belongs to the conserved Brix domain protein family ${ }^{[20]}$. The PPAN protein exists in the nucleolus and is linked to ribosome biogenesis. In addition, the PPAN protein also exists in mitochondria, and the knockdown of the PPAN gene can trigger p53-independent mitochondrial apoptosis and nucleolar stress ${ }^{[21]}$. In 2003, STK40 was identified as a new SINK-homologous serine/threonine protein kinase, which could inhibit TNF-induced NF-KB activation $^{[22]}$. Maubant et al. believed that STK40 was highly expressed in triple-negative breast cancers (TNBCs). STK40 depletion induces the apoptosis of TNBC cells, leading to tumor cell proliferation and clonal formation ${ }^{[23]}$. In addition, STK40 can participate in the activation of the MAPK/ extracellular signalregulated kinase pathway and interact with the E3 ubiquitin ligase constitutive photomorphogenic protein $1^{[24-26]}$. SNORD16 is highly expressed in colon cancer and can promote the growth, proliferation, migration, and invasion of colon cancer cells. The expression of SNORD16 is an independent prognostic factor, and patients with high expression usually have a worse prognosis ${ }^{[27]}$.

Our study also had some limitations. First, not many tumor samples contained immunotherapy response information in the shared database. Therefore, it is necessary to further expand the sample size for full 
verification. Second, our study was based on transcriptome data, and the transcription level is not exactly the same as the protein level. Third, transcriptome data mainly contains protein-coding genes, but the role of noncoding RNA in tumors cannot be ignored ${ }^{[28]}$. Finally, the study lacked detailed mechanistic research. These limitations will be resolved in future studies.

\section{Conclusions}

In conclusion, we established an anti-PD1 immunotherapy response score prediction model for patients with melanoma. The model showed good predictive power and helped to identify people who benefit from anti-PD1 immunotherapy to improve the prognosis.

\section{Methods}

\section{Materials and Methods}

\section{Data source}

The melanoma transcriptome datasets (GSE78220 and GSE91061) and the renal cell carcinoma transcriptome dataset (GSE67501) containing anti-PD1 immunotherapy response information from the GEO database were obtained. In the GSE78220 dataset, 15 samples responded to anti-PD1 immunotherapy (complete response (CR)/partial response (PR)) and 13 samples did not respond (progressed disease (PD)/stable disease (SD)). In the GSE91061 dataset, 23 samples responded to antiPD1 immunotherapy (CR/PR) and 82 samples did not respond (PD/SD). In the GSE67501 dataset, 4 samples responded to anti-PD1 immunotherapy (CR/PR) and 7 samples did not respond (PD/SD).

\section{Statistical analysis}

We used the "ComBat" package to perform batch calibration and merged the two datasets (GSE78220 and GSE91061) into a new dataset. Differentially expressed genes of the new dataset were obtained by performing differential expression analysis using the "limma" package $(P<0.05 ; \mid \log$ (Fold change) $\mid>1)$. We presented differentially expressed genes in the form of heat maps using the "pheatmap" package. The Wilcoxon test was used to analyze the differences between the two groups. We used the "caret" package to randomly divide the new data set into training and validation data sets according to the ratio of the number of samples to $6: 4$ for constructing and verifying the immunotherapy response prediction model. Lasso regression and cross-validation were performed using the "glmnet" package to construct a binary logistic regression model for predicting the response of patients with melanoma with anti-PD1 immunotherapy. We used the "pROC" package to perform receiver operating characteristic (ROC) curve analysis so as to evaluate the effectiveness of the model and related indicators. Principal component analysis (PCA) was performed using the "factoextra" package to achieve the purpose of comprehensive evaluation. 
We used the "estimate" package to estimate the immune component and matrix component contents of all samples in the tumor microenvironment in the entire dataset. Gene set enrichment analysis (GSEA) was used to illustrate the biological process of enrichment of differentially expressed genes between the high- and low-response score groups. Kyoto Encyclopedia of Genes and Genomes (KEGG), Gene ontology (GO), Biocarta, and Hallmark were downloaded from the MSigDB database as the reference gene sets for GSEA analysis. Only enrichment pathways with NOM $P<0.05$ were considered meaningful. The CIBERSORT algorithm was used to estimate the abundance of tumor-infiltrating immune cells (TICs) in tumor samples; only tumor samples with $P<0.05$ were selected. The correlation between TICs was assessed by Pearson correlation. A $P$ value $<0.05$ indicated a statistically significant difference. All statistical analyses were performed using the $\mathrm{R}$ language.

\section{Abbreviations}

PD1: programmed cell death 1; MAPK: mitogen activated protein kinase; GEO: Gene Expression Omnibus; CR: complete response; PR: partial response; PD: progressed disease; SD: stable disease; ROC: receiver operating characteristic; PCA: principal component analysis; GSEA: gene set enrichment analysis; KEGG: Kyoto Encyclopedia of Genes and Genomes; GO: Gene ontology; TICs: tumor-infiltrating immune cells; PTPDC1: protein tyrosine phosphatase domain containing 1; SDCCAG8: serologically defined colon cancer antigen 8, PPAN: peter pan homolog; STK40: serine/threonine kinase 40; SNORD16: and small nucleolar RNA, C/D box 16; AUC: area under the curve; SE: sensitivity, SP: specificity; PPV: positive predictive value; NPV: negative predictive value; PDL1: programmed death ligand 1; dMMR: deficient mismatch repair; MLH1: mutL homologue 1; PMS2: postmeiotic segregation increased 2; MSH2: mutS homologue 2; MSH6: mutS homologue 6.

\section{Declarations}

\section{Acknowledgements}

We thank the staff of GSE78220, GSE91061 and GSE67501 for their hard work. The data they upload is an important basis for this research.

\section{Authors' Contributions:}

Xiaoyan Li: conceptualization and writing - original draft; Guoyin Li: supervision, writing - review and editing; Xiyang Tang: supervision, writing - review and editing; Yongsheng Zhou: writing - review and editing; Kaifu Zheng: writing - review and editing; Zhonglin Luo: writing - review and editing; Xiao Zhang: writing - review and editing; Yanlu Xiong: supervision, writing - review and editing, and funding acquisition; Yangbo Feng: supervision, writing - review and editing, and formal analysis. 


\section{Funding:}

This study was funded by the National Natural Science Foundation of China (82002421) and the Young Talent Program of Tangdu.

\section{Availability of data and materials}

The datasets $₫$ GSE78220, GSE91061 and GSE67501) involved in this article are deposited in the GEO (www.ncbi.nlm.nih. gov/geo).

\section{Ethics approval and consent to participate}

Not applicable.

\section{Consent for publication}

Not applicable

\section{Competing interests:}

The authors have no conflicts of interest to declare.

\section{Author details}

${ }^{1}$ Department of blood transfusion, Shanxi Provincial People's Hospital ,Taiyuan, Shanxi, 030012, China.

${ }^{2}$ College of Life Science and Agronomy, Zhoukou Normal University, Zhoukou, 466000, China. ${ }^{3}$ Academy of medical science, Zhengzhou University, Zhengzhou, 450001, China. ${ }^{4}$ Department of Thoracic Surgery, Tangdu Hospital, Fourth Military Medical University, Xi'an, Shaanxi, 710038, China. ${ }^{5}$ Department of Cardiothoracic Surgery, Peace Hospital affiliated to Changzhi Medical College『Changzhi Medical College, Shanxi,046000, China. ${ }^{6}$ State Key Laboratory of Cancer Biology, Department of Biochemistry and Molecular Biology, Fourth Military Medical University, Xi'an, Shaanxi, 710038, China.

\section{References}

1. C Karimkhani, Green A C, Nijsten T, Weinstock M A, Dellavalle R P, Naghavi M, Fitzmaurice C. The global burden of melanoma: results from the Global Burden of Disease Study 2015[J]. Br J Dermatol, 2017, 177(1): 134-140. 
2. S J Henley, Ward E M, Scott S, Ma J, Anderson R N, Firth A U, Thomas C C, Islami F, Weir H K, Lewis D R, Sherman R L, Wu M, Benard V B, Richardson L C, Jemal A, Cronin K, Kohler B A. Annual report to the nation on the status of cancer, part I: National cancer statistics[J]. Cancer, 2020, 126(10): 22252249.

3. Y Wu, Wang Y, Wang L, Yin P, Lin Y, Zhou M. Burden of melanoma in China, 1990-2017: Findings from the 2017 global burden of disease study[J]. Int J Cancer, 2020, 147(3): 692-701.

4. Chinese guidelines for diagnosis and treatment of melanoma 2018 (English version)[J]. Chin $\mathrm{J}$ Cancer Res, 2019, 31(4): 578-585.

5. B D Curti, Faries M B. Recent Advances in the Treatment of Melanoma[J]. N Engl J Med, 2021, 384(23): 2229-2240.

6. R W Jenkins, Fisher D E. Treatment of Advanced Melanoma in 2020 and Beyond[J]. J Invest Dermatol, 2021, 141(1): 23-31.

7. V Saranga-Perry, Ambe C, Zager J S, Kudchadkar R R. Recent developments in the medical and surgical treatment of melanoma[J]. CA: A Cancer Journal for Clinicians, 2014, 64(3): 171-185.

8. J Larkin, Chiarion-Sileni V, Gonzalez R, Grob J J, Rutkowski P, Lao C D, Cowey C L, Schadendorf D, Wagstaff J, Dummer R, Ferrucci P F, Smylie M, Hogg D, Hill A, Marquez-Rodas I, Haanen J, Guidoboni M, Maio M, Schoffski P, Carlino M S, Lebbe C, Mcarthur G, Ascierto P A, Daniels G A, Long G V, Bastholt L, Rizzo J I, Balogh A, Moshyk A, Hodi F S, Wolchok J D. Five-Year Survival with Combined Nivolumab and Ipilimumab in Advanced Melanoma[J]. N Engl J Med, 2019, 381(16): 1535-1546.

9. O Hamid, Robert C, Daud A, Hodi F S, Hwu W J, Kefford R, Wolchok J D, Hersey P, Joseph R, Weber J S, Dronca R, Mitchell T C, Patnaik A, Zarour H M, Joshua A M, Zhao Q, Jensen E, Ahsan S, Ibrahim N, Ribas A. Five-year survival outcomes for patients with advanced melanoma treated with pembrolizumab in KEYNOTE-001[J]. Ann Oncol, 2019, 30(4): 582-588.

10. C Robert, Grob J J, Stroyakovskiy D, Karaszewska B, Hauschild A, Levchenko E, Chiarion Sileni V, Schachter J, Garbe C, Bondarenko I, Gogas H, Mandala M, Haanen J, Lebbe C, Mackiewicz A, Rutkowski P, Nathan P D, Ribas A, Davies M A, Flaherty K T, Burgess P, Tan M, Gasal E, Voi M, Schadendorf D, Long G V. Five-Year Outcomes with Dabrafenib plus Trametinib in Metastatic Melanoma[J]. N Engl J Med, 2019, 381(7): 626-636.

11. E E Patton, Mueller K L, Adams D J, Anandasabapathy N, Aplin A E, Bertolotto C, Bosenberg M, Ceol C J, Burd C E, Chi P, Herlyn M, Holmen S L, Karreth F A, Kaufman C K, Khan S, Kobold S, Leucci E, Levy C, Lombard D B, Lund A W, Marie K L, Marine J C, Marais R, Mcmahon M, Robles-Espinoza C D, Ronai Z A, Samuels Y, Soengas M S, Villanueva J, Weeraratna A T, White R M, Yeh I, Zhu J, Zon L I, Hurlbert M S, Merlino G. Melanoma models for the next generation of therapies[J]. Cancer Cell, 2021, 39(5): 610-631.

12. S Ganesan, Mehnert J. Biomarkers for Response to Immune Checkpoint Blockade[J]. Annual Review of Cancer Biology, 2020, 4(1): 331-351.

13. E M Jacobi, Landon G, Broaddus R R, Roy-Chowdhuri S. Evaluating Mismatch Repair/Microsatellite Instability Status Using Cytology Effusion Specimens to Determine Eligibility for Immunotherapy[J]. 
Arch Pathol Lab Med, 2021, 145(1): 46-54.

14. D Schadendorf, Van Akkooi A C J, Berking C, Griewank K G, Gutzmer R, Hauschild A, Stang A, Roesch A, Ugurel S. Melanoma[J]. The Lancet, 2018, 392(10151): 971-984.

15. S Roychowdhury, Chinnaiyan A M. Translating cancer genomes and transcriptomes for precision oncology[J]. CA Cancer J Clin, 2016, 66(1): 75-88.

16. D Hanahan, Weinberg R A. Hallmarks of cancer: the next generation[J]. Cell, 2011, 144(5): 646-674.

17. D H Zineldeen, Shimada M, Niida H, Katsuno Y, Nakanishi M. Ptpcd-1 is a novel cell cycle related phosphatase that regulates centriole duplication and cytokinesis[J]. Biochem Biophys Res Commun, 2009, 380(3): 460-466.

18. R Airik, Slaats G G, Guo Z, Weiss A C, Khan N, Ghosh A, Hurd T W, Bekker-Jensen S, Schroder J M, Elledge S J, Andersen J S, Kispert A, Castelli M, Boletta A, Giles R H, Hildebrandt F. Renal-retinal ciliopathy gene Sdccag8 regulates DNA damage response signaling[J]. J Am Soc Nephrol, 2014, 25(11): 2573-2583.

19. J Huang, Ji E H, Zhao X, Cui L, Misuno K, Guo M, Huang Z, Chen X, Hu S. Sox11 promotes head and neck cancer progression via the regulation of SDCCAG8[J]. J Exp Clin Cancer Res, 2019, 38(1): 138.

20. J C Migeon, Garfinkel M S, Edgar B A. Cloning and characterization of peter pan, a novel Drosophila gene required for larval growth[J]. Mol Biol Cell, 1999, 10(6): 1733-1744.

21. A S Pfister, Keil M, Kuhl M. The Wnt Target Protein Peter Pan Defines a Novel p53-independent Nucleolar Stress-Response Pathway[J]. J Biol Chem, 2015, 290(17): 10905-10918.

22. J Huang, Teng L, Liu T, Li L, Chen D, Li F, Xu L-G, Zhai Z, Shu H-B. SINK[J]. Biochemical and Biophysical Research Communications, 2003, 309(4): 774-778.

23. S Maubant, Tahtouh T, Brisson A, Maire V, Nemati F, Tesson B, Ye M, Rigaill G, Noizet M, Dumont A, Gentien D, Marty-Prouvost B, De Koning L, Mahmood S F, Decaudin D, Cruzalegui F, Tucker G C, Roman-Roman S, Dubois T. LRP5 regulates the expression of STK40, a new potential target in triplenegative breast cancers[J]. Oncotarget, 2018, 9(32): 22586-22604.

24. I Durzynska, Xu X, Adelmant G, Ficarro S B, Marto J A, Sliz P, Uljon S, Blacklow S C. STK40 Is a Pseudokinase that Binds the E3 Ubiquitin Ligase COP1[J]. Structure, 2017, 25(2): 287-294.

25. L Li, Sun L, Gao F, Jiang J, Yang Y, Li C, Gu J, Wei Z, Yang A, Lu R, Ma Y, Tang F, Kwon S W, Zhao Y, Li J, Jin Y. Stk40 links the pluripotency factor Oct4 to the Erk/MAPK pathway and controls extraembryonic endoderm differentiation[J]. Proc Natl Acad Sci U S A, 2010, 107(4): 1402-1407.

26. H Yu, He K, Li L, Sun L, Tang F, Li R, Ning W, Jin Y. Deletion of STK40 protein in mice causes respiratory failure and death at birth[J]. J Biol Chem, 2013, 288(8): 5342-5352.

27. J Y He, Liu X, Qi Z H, Wang Q, Lu W Q, Zhang Q T, He S Y, Wang Z D. Small Nucleolar RNA, C/D Box 16 (SNORD16) Acts as a Potential Prognostic Biomarker in Colon Cancer[J]. Dose Response, 2020, 18(2): 1559325820917829.

28. H Ling, Fabbri M, Calin G A. MicroRNAs and other non-coding RNAs as targets for anticancer drug development[J]. Nat Rev Drug Discov, 2013, 12(11): 847-865. 


\section{Tables}

Due to technical limitations, tables are only available as a download in the Supplemental Files section.

Figures
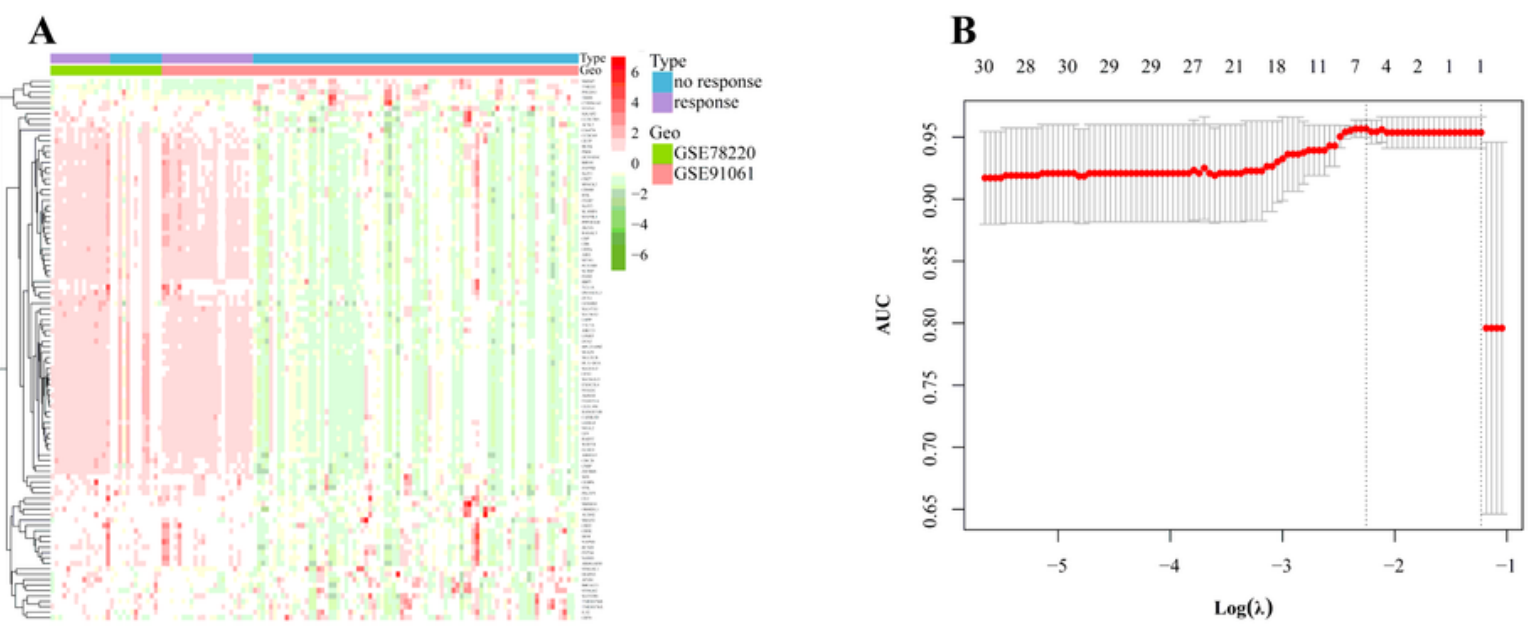

C

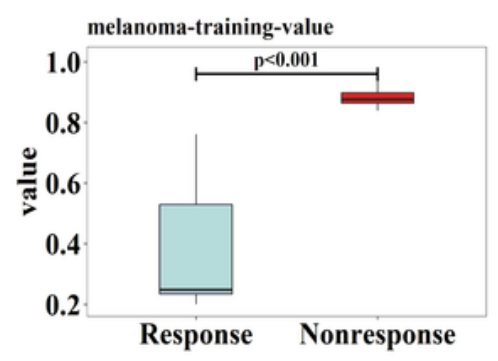

D

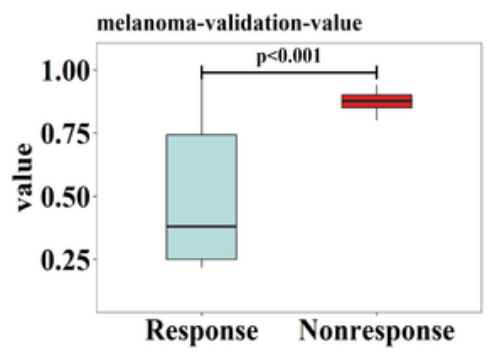

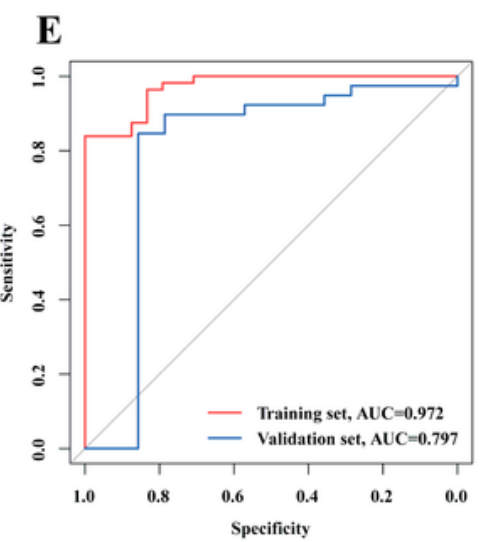

Figure 1 
Construction and evaluation of the predictive model of anti-PD1 immunotherapy response in patients with melanoma. (A) Top 100 differentially expressed genes in a merged dataset. (B) Key genes obtained by performing Lasso regression and cross-validation. (C-D) The relationship between the actual immunotherapy response of tumor samples and the immunotherapy response score in the training and validation datasets. (E) The AUC of the immunotherapy response score prediction model based on the training and validation datasets.
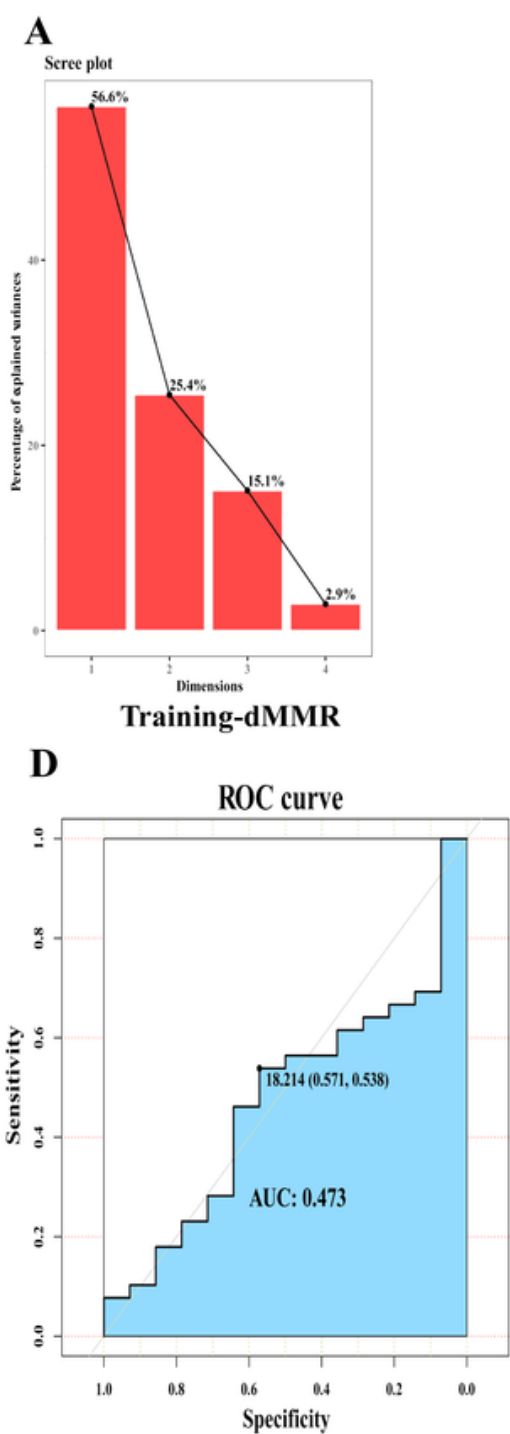

Validation-dMMR
B

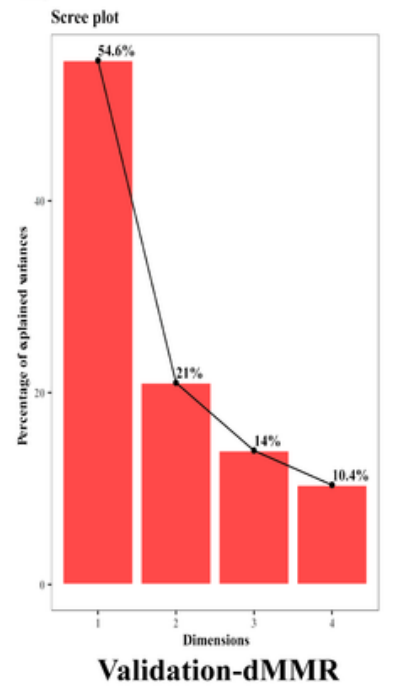

E

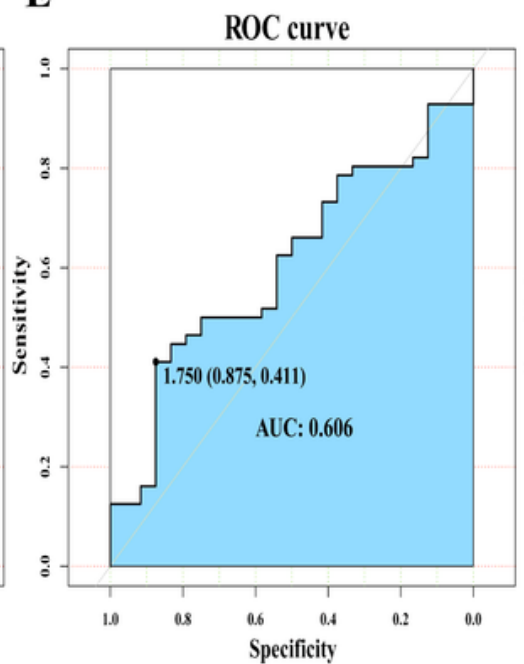

Training-PDL1

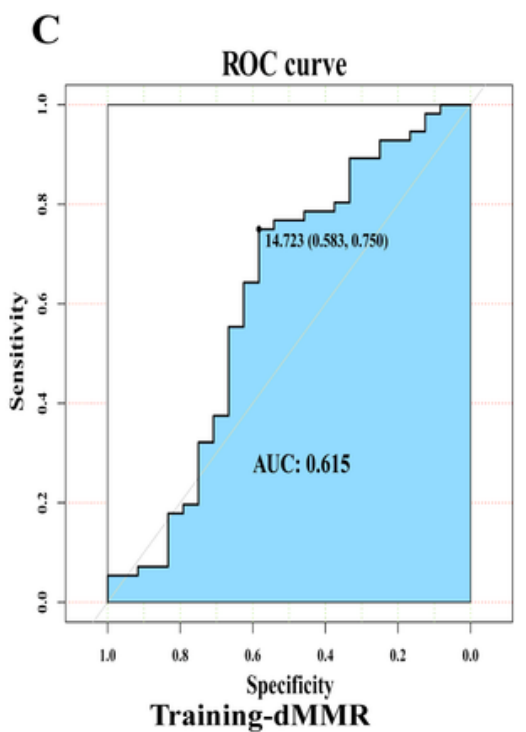

$\mathbf{F}$

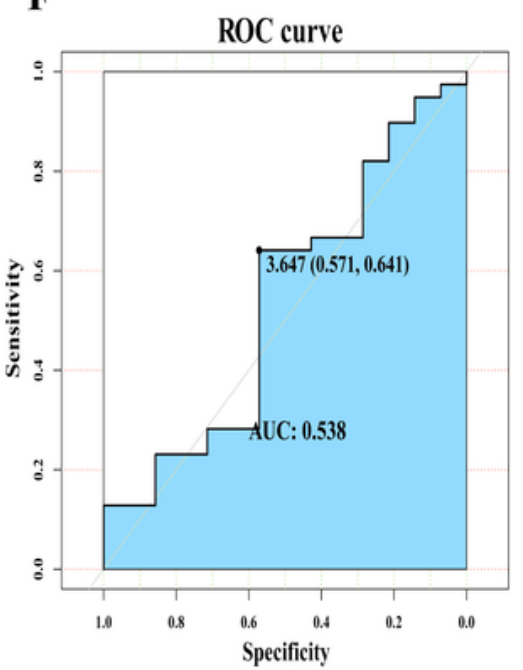

Validation-PDL1 
The accuracy of common predictors of immunotherapy efficacy in patients with melanoma. (A-B) Comprehensive evaluation of genes related to DNA mismatch repair in the training and validation datasets by PCA. (C-D) The AUC of the dMMR algorithm in the training and validation datasets. (E-F) The AUC of the PDL1 expression in the training and validation datasets.

A

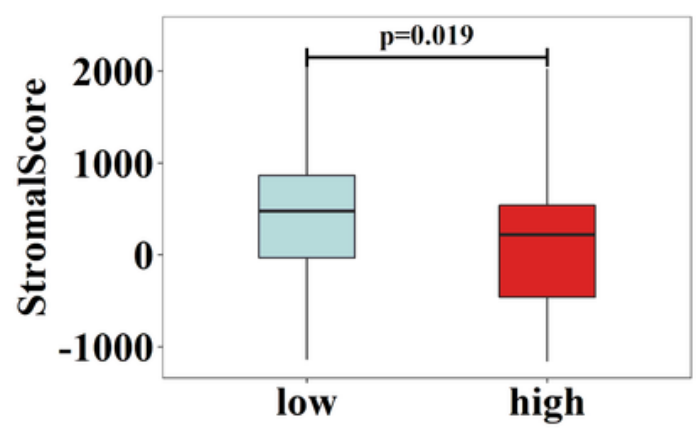

C

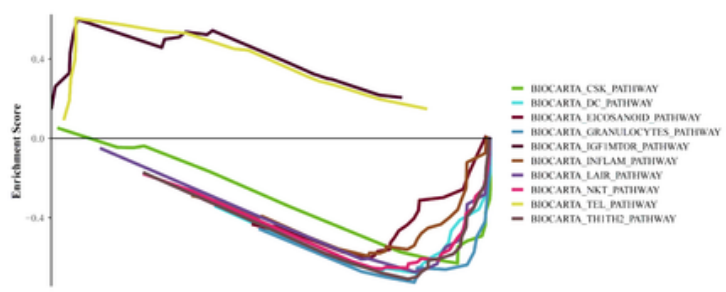

$\mathbf{E}$
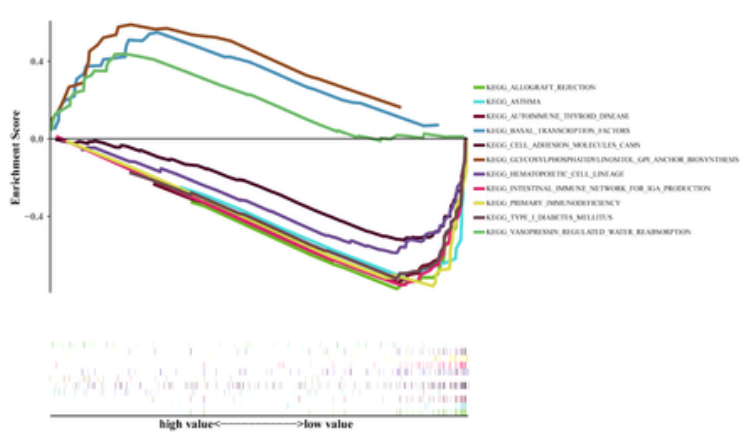

B

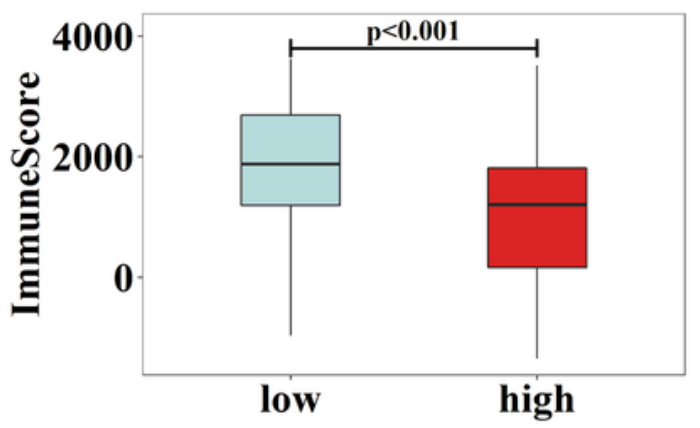

D
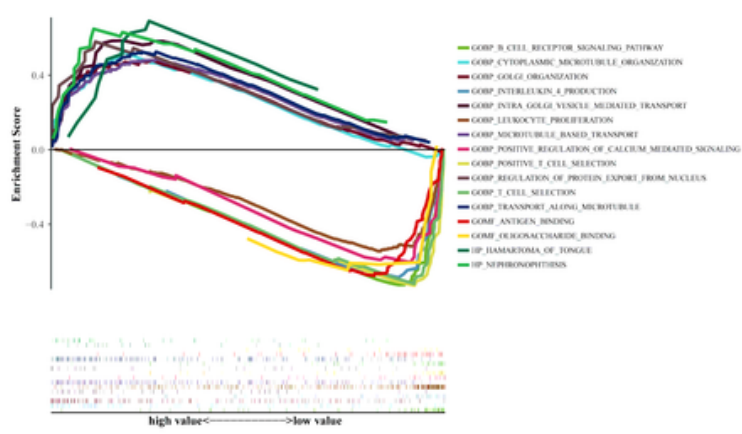

\section{$\mathbf{F}$}
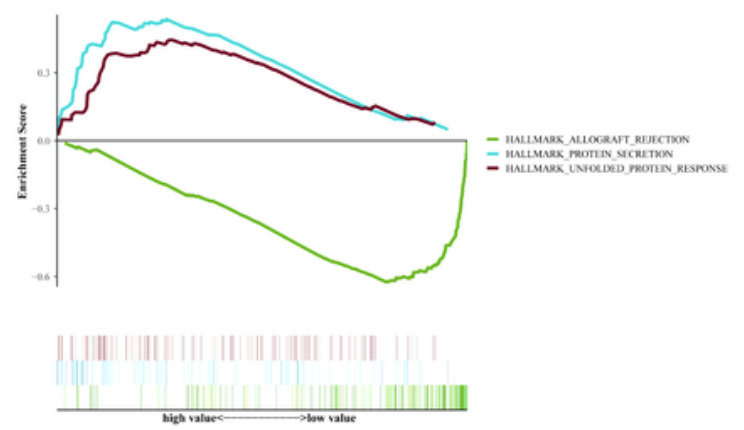

Figure 3 
Alterations of immune and matrix components and signaling in high- and low-score groups. (A-B) Correlation between the ratio of matrix and immune components in tumor samples and the score of immunotherapy response. (C-F) Alterations of signaling in high- and low-score groups.

A

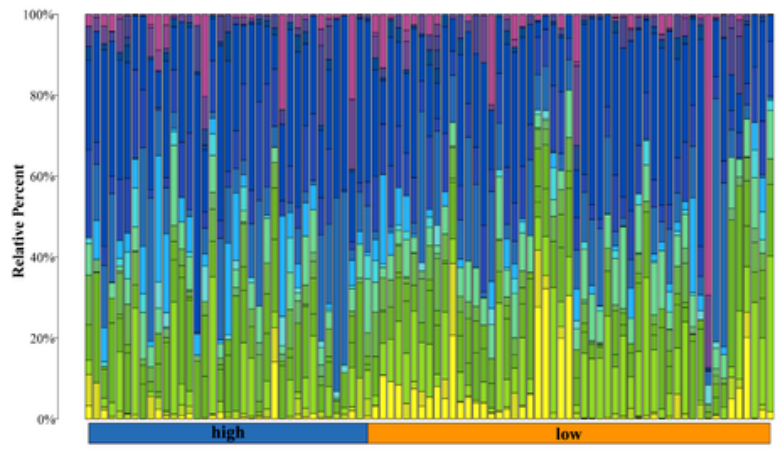

C

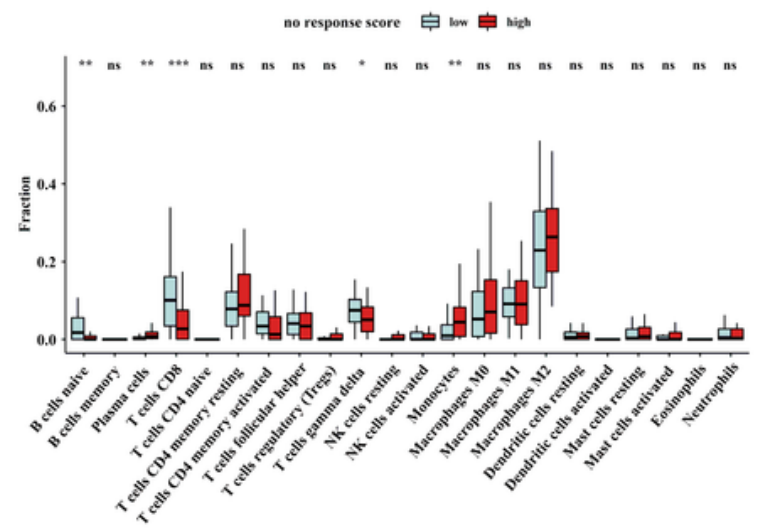

E

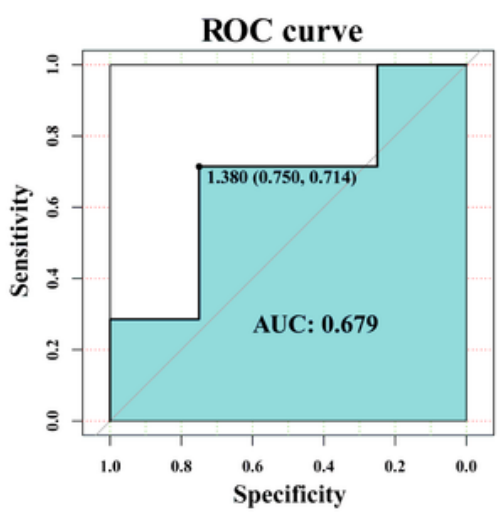

B
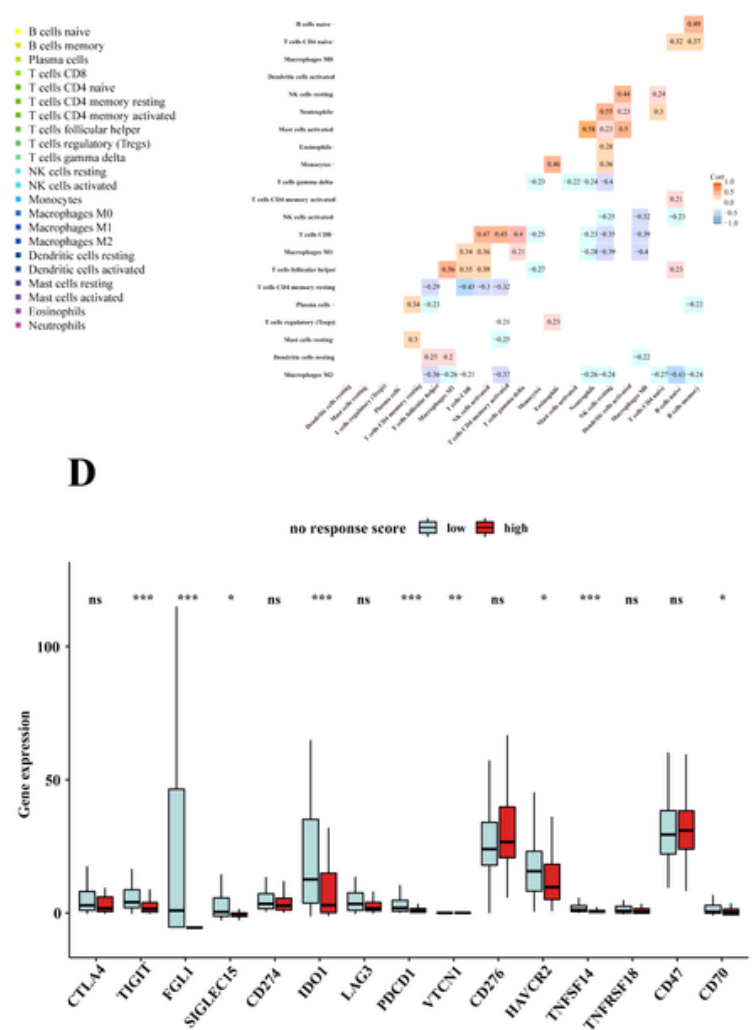

$\mathbf{F}$

GSE67501-value

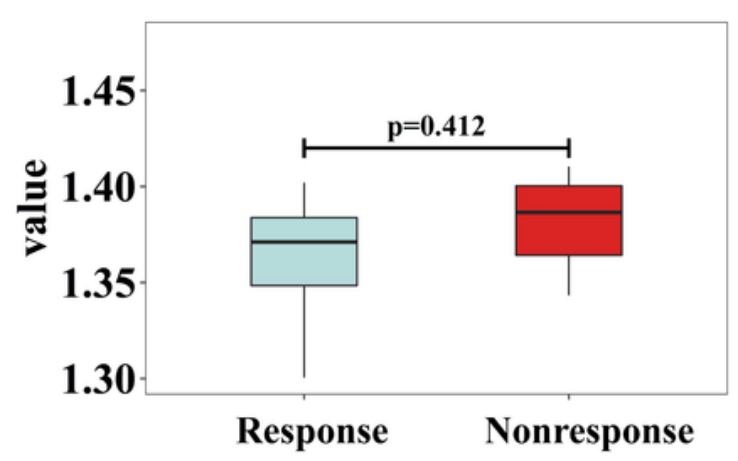

Figure 4

Correlation between the score of immunotherapy response and tumor microenvironment. (A) Expression profiles of 22 immune cells in melanoma tissues. (B) Correlation analysis of immune cells in melanoma 
tissues. (C) Difference existed in the expression of immune cells between the high and low scores of immunotherapy responses. (D) Differences in the expression of common immune checkpoint genes between the high and low scores of immunotherapy responses. (E) The AUC of the immunotherapy response score in the renal cell carcinoma dataset GSE67501. (F) Difference in the immunotherapy response score between the immunotherapy response group and the nonresponse group.

\section{Supplementary Files}

This is a list of supplementary files associated with this preprint. Click to download.

- Table2.xlsx

- Table3.xlsx

- Table1.xlsx 